\title{
Remote Estimation of Intra-Parcel Grapes Quantity From Three-Dimensional Imagery Technique Using Ground-based Microwave FMCW Radar
}

\author{
Dominique Henry, Hervé Aubert, Thierry Véronèse and Éric Serrano
}

\begin{abstract}
For better benefits and yields, a good estimation of grapes quantity in vineyard is necessary. In this paper, three-dimensional (3D) imagery technique using conventional $24 \mathrm{GHz}$ Frequency-Modulated Continuous-Wave (FMCW) Radar is applied for detecting and remotely estimating the intra-parcel quantity of grapes. An estimation is possible even in presence of natural or artificial clutters such as leaves, wood or irrigation hoses. The microwave sensing is performed from the radar beam scanning of a vineyard and an estimator is defined to derive the quantity of grapes in grapevines from radar echoes distribution in the interrogated 3D scene. An algorithm based on contour detection is applied to the 3D radar image and a new parameter, called the spread factor, is defined for classifying the echo levels of grapes. The quantity of grapes is finally deduced from an appropriate estimator. This remote sensing approach brings a new and flexible solution for precision viticulture by estimating the grape quantity even for grapes hidden by leaves.
\end{abstract}

\section{Introduction}

Nowadays precision agriculture (PA) is mandatory to optimize crop yields, particularly for very large field areas. It requires a well understanding of crop science and environmental impacts. When it concerns specifically the study of vineyards, the PA refers to precision viticulture (PV). Two main technologies applied to PV can be identified: (a) variable-rate-technologies (VRT) which use agricultural machines controlled by data from sensors and Global Positioning Systems (GPS) and, (b) monitoring technologies which focus on the observation of biological or structural parameters such as the leaf area index (LAI) [1, 2], the canopy and vigour (that is, leaf area and pruning weight), the chlorophyll content or/and the anthocyanin concentration [3], the sugar content [4] or the soil properties [5]. Satellites [1,2], airborne [6] or un-manned aerial vehicles (drones) are also solutions for multi-parcels and large areas monitoring while sensors based on ground, such as low frequency Ground-Penetrating Radar (GPR) [7], LiDAR [8] or optical cameras [9], are good candidates for monitoring the LAI, the vigour or the ground electrical conductivity.

The major challenge addresses in this paper is to monitor the quantity of grapes on grapevines. Be able to know the volume of grapes in advance, that is, before the crop, offers many economic advantages: (a) it allows predicting the appropriate quantity of materials to rent several months before the grapes harvesting and, (b) it could estimate grapes losses to be fairly compensated by insurances in case of severe weather conditions. Grapes detection has already been investigated mostly from optical technologies (see, e.g., [9]). The main drawback of such approach is that grapes may be partially or completely hidden by leaves or wood and consequently they cannot be easily detected by optical sensors. Moreover volume estimation of grapes is difficult since only 2D images are available from such sensors.

In this paper the objective is to estimate the volume of grapes on grapevines using a groundbased Frequency Modulated Continuous Wave (FMCW) radar operating at $24 \mathrm{GHz}$. Compared to optical systems, the microwave radar technology allows remotely sensing the scene in depth and 
detecting most hidden grapes. Microwave frequencies, especially Super High Frequencies (3 to 30 $\mathrm{GHz}$ ), are advantageously used here for grapes quantity estimation since the water in the grapes is highly reflective at these frequencies. We show here that the estimation of grapes volume can be derived from the proposed microwave sensing even in presence of natural or artificial clutters such as leaves, wood and irrigation hoses. The sensing is performed from the radar beam scanning of a vineyard. An appropriate estimator is proposed for deriving the quantity of grapes in grapevines from radar echoes distribution in the 3D scene.

\section{Description of the measurement setup}

A $24 \mathrm{GHz}$ FMCW radar used in the experiment generates a frequency-modulated signal with a carrier frequency $f_{0}=23.8 \mathrm{GHz}$ and a bandwidth $B=2 \mathrm{GHz}$. The bandwidth is a crucial parameter leading directly to the theoretical depth resolution $d=\frac{c}{2 B}=7.5 \mathrm{~cm}$ where $c$ denotes the celerity of light in vacuum. The signal is transmitted using a horn lens antenna (Tx antenna) with a gain of 28 $\mathrm{dBi}$ and a beamwidth of $6^{\circ}$. Electromagnetic waves backscattered by the grapevines are received by a $1 \times 5$ patch array antenna (Rx antenna) with a gain of $8.6 \mathrm{dBi}$ and a beamwidth of $60^{\circ}$ in azimuth and $25^{\circ}$ in elevation. The backscattered signal is received by the radar antenna and is converted into a beat frequency spectrum which gives the echo level of the grapevine in a given direction as a function of the interrogation range. The power transmitted by the radar front-end is of $20 \mathrm{dBm}$ $(100 \mathrm{~mW})$. The radar and antennas are mounted on a mechanical platform controlled by a computer unit (Fig. 1). The system performs a rotation of the Rx and Tx radar antennas with an accuracy of $1^{\circ}$ in azimuth $(\theta)$ and in elevation $(\varphi)$. This rotation allows performing the mechanical scanning of the radar beam and the $3 \mathrm{D}$ representation of the grapevine echo level distribution in the interrogated 3D scene can be derived.

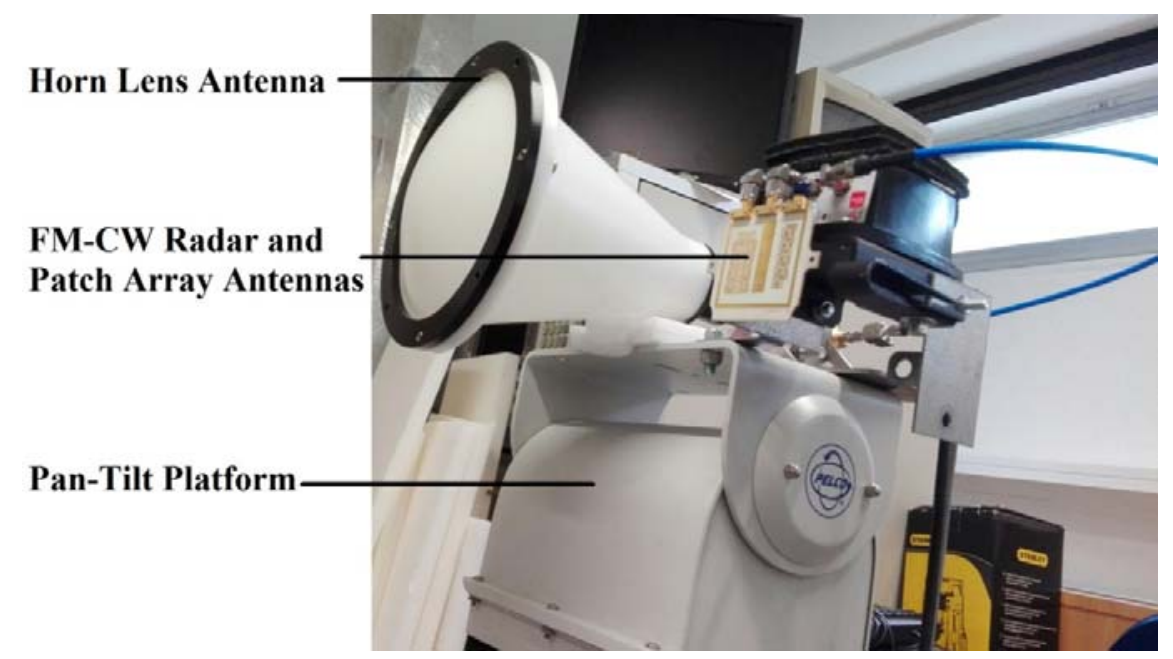

Fig 1. Ground-based microwave FMCW radar proposed for precision viticulture 


\section{Vineyard clutter response}

3D Radar images are taken in a vineyard in Gaillac, France. The microwave FMCW radar is ground-based and moves between rows of grape vines. A beam scanning is performed in front of each plant at a distance of $1.0 \mathrm{~m}$ with angles of $\pm 30^{\circ}$ in azimuth and $-10^{\circ}$ to $30^{\circ}$ in elevation. This scanning allows measuring the beat frequency spectrum in 2501 directions in interrogated scene. As an illustration, the cut plane of obtained radar image is shown on Fig. 2. Vine rows are easily detectable in this image because of their specific geometrical and physical properties. Rows are separated by a distance of $2.5 \mathrm{~m}$ while the separation distance between grapevine plants is of 1 $\mathrm{m}$. These plants are composed of leaves, grapes and wood. The water inside grapes and leaves has a relative permittivity around 30 at a frequency of $24 \mathrm{GHz}$ and generates high reflection of the incident microwave field. Non-organic clutters (such as iron wires or irrigation hoses) create also strong electromagnetic echoes.

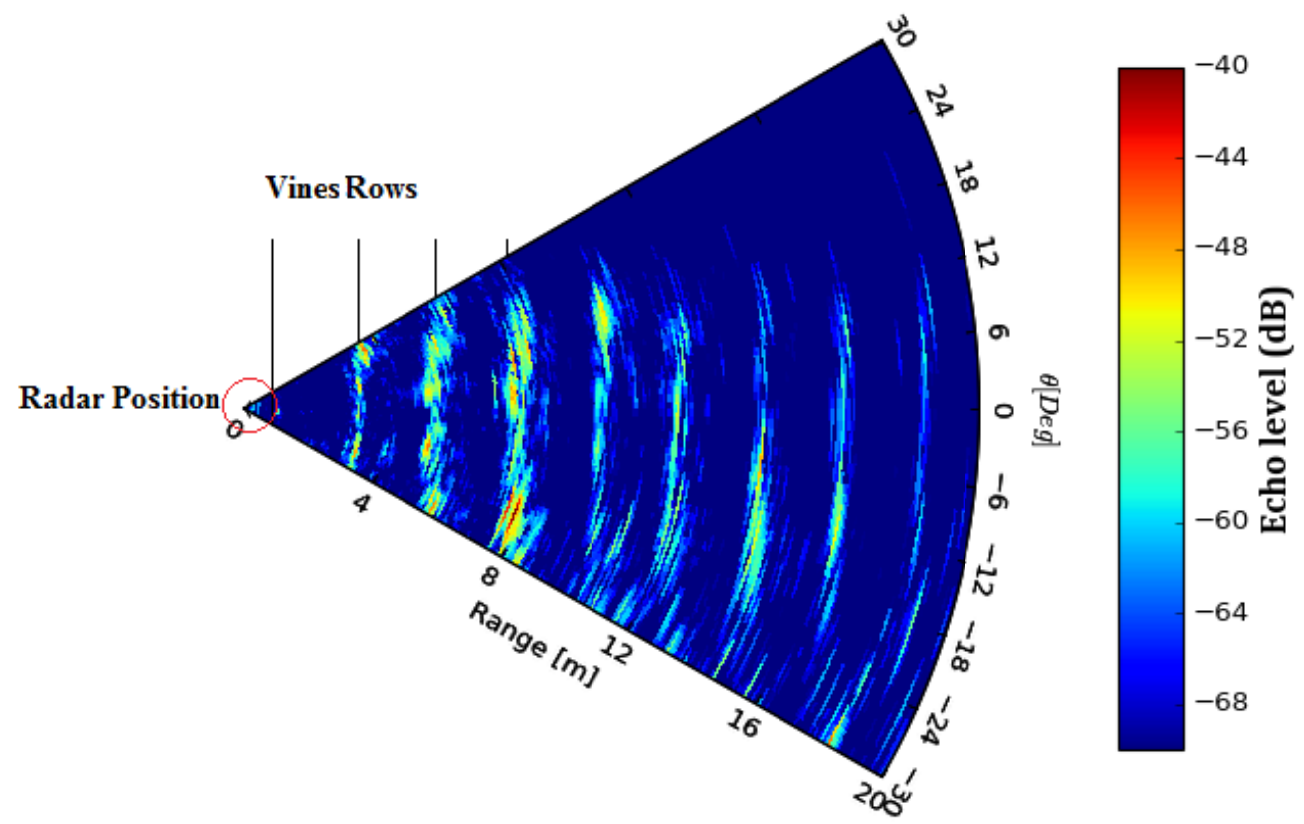

Fig 2. Two-dimensional polar plot of electromagnetic echoes from the vineyard (cut-plane $\left.\varphi=0^{\circ}\right)$. High echo levels allows locating the vines rows.

\section{D scanning of grapevine plants}

For the estimation of grapes volume, the electromagnetic echo levels is measured from $0.5 \mathrm{~m}$ to $1.5 \mathrm{~m}$ in front of various grapevine plants. Two varieties of grapes are studied here: Gamay and Black Manseng (Fig. 3). Radar measurement of three plants of each variety is performed before and after the grapes crop. As observed in Fig.3, these varieties have many leaves and consequently, a detection of all grapes from optical sensors is highly problematic while the microwave interrogation allows sensing in depth the plants and detecting hidden grapes. For fixed volumetric mass density of grapes, the grape volume increases linearly with the mass and the water content. Consequently an estimation of the volume of grapes on a plant can be derived from the measurement of the echo level generated by the water content in grapes. 


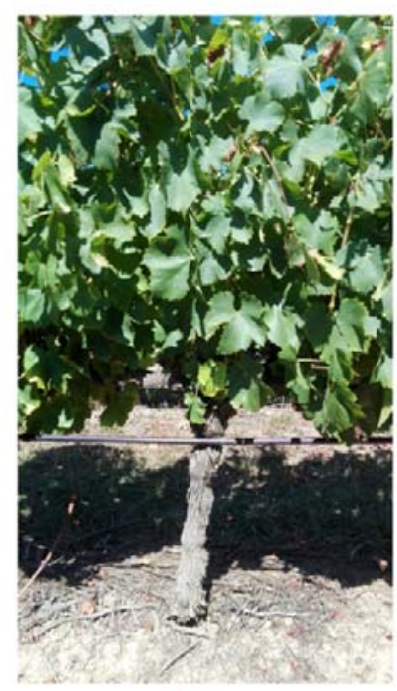

(a)

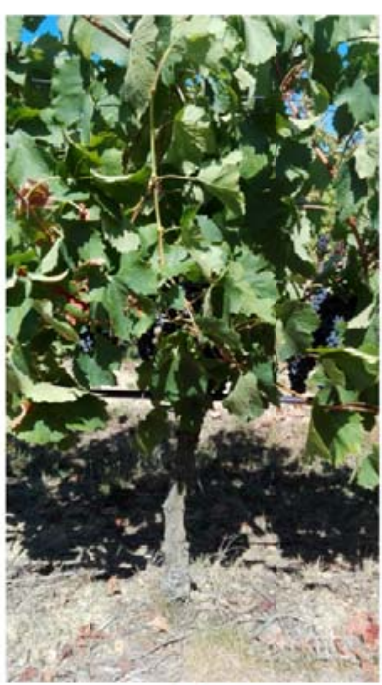

(b)

Fig 3. Grapevines of (a) Gamay and (b) Black Manseng.

The measured data from the 3D radar beam scanning are originally obtained in spherical coordinates. A Cartesian conversion is applied and 93324 voxels are then derived for each grapevine. The resulting volume resolution depends on the size of the voxel $s$ defined by:

$$
s=d_{x} \times d_{y} \times d
$$

where $d_{x}$ and $d_{y}$ denote respectively the $\mathrm{x}$ and y cross-range resolutions defined by the interpolation during the coordinates conversion. Here $d_{x}=d_{y}=1.75 \mathrm{~cm}$ and consequently, the volume resolution is $s=23 \mathrm{~cm}^{3}$. Fig. 4 shows the resulting 3D scanning of a Gamay grapevine plant. The echo levels are displayed by using isosurfaces which represent layers of echo levels sharing the same value. These layers are stacked together and provide a convenient way to display the 3D distribution of echoes. It can be observed that regions with higher echo levels are located near leaves and grapes above a height of around $60 \mathrm{~cm}$. The trunk is also easily detectable at $\mathrm{x}=0 \mathrm{~m}$ with a height below 60 $\mathrm{cm}$. The identification of regions containing leaves and grapes is not easy at first glance. However the analysis reported in the next section will allow estimating the volume of the grapes in the plant. 


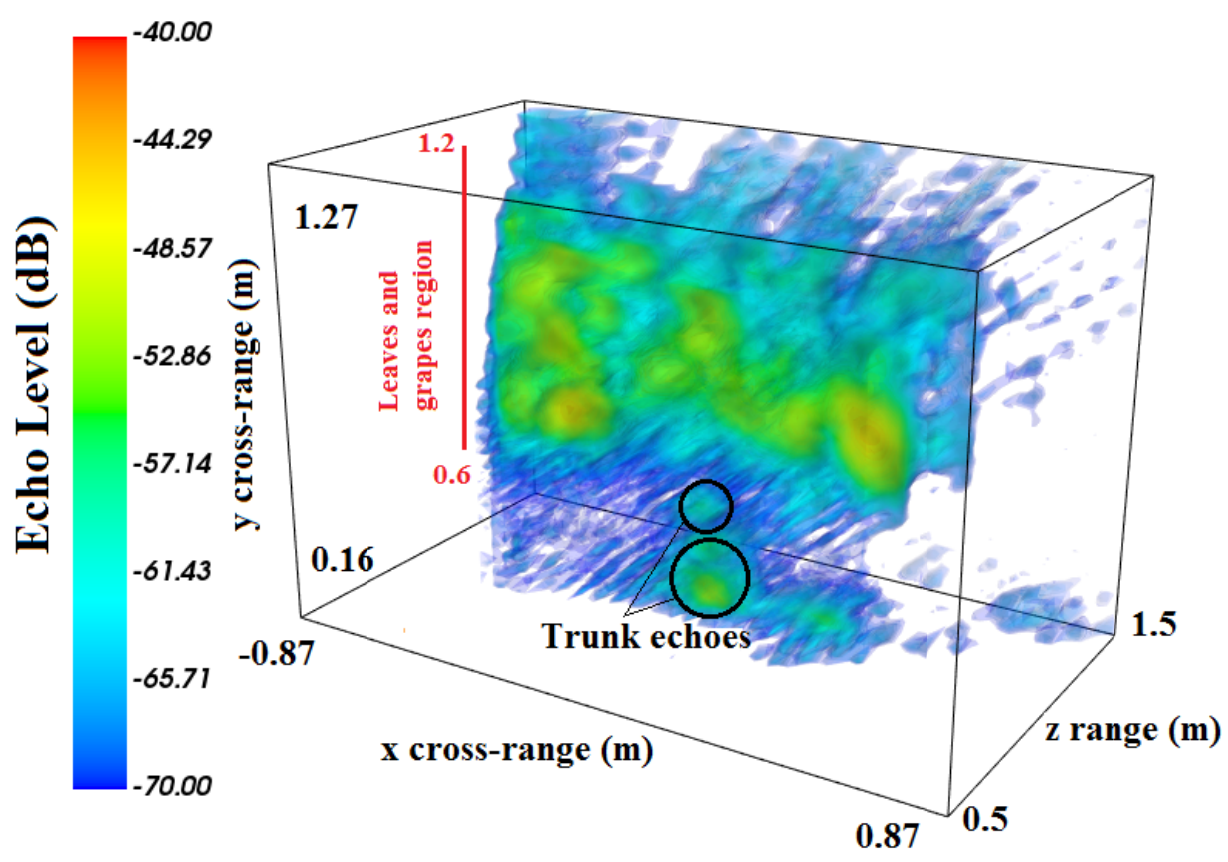

Fig 4. Three-dimensional representation of echo level distribution of a Gamay grapevine. The region of height comprised between $0.6 \mathrm{~m}$ and $1.2 \mathrm{~m}$ corresponds to the location of grapes, wood and leaves

\section{Grapes quantity estimation}

In order to estimate the quantity of grapes from the measured radar echoes distribution in the interrogated 3D scene, the analysis of each echo level generated by the clutter is performed. The objective is to determine if echoes are due to the electromagnetic backscattering by grapes or by the clutter such as, e.g., the leaves, trunk, wood or iron wire. Knowing that grapes grow at height between $60 \mathrm{~cm}$ to $90 \mathrm{~cm}$ from the ground, the proposed analysis considers only echoes located between these two heights. For classifying these selected echoes, a standard marching squares contour algorithm is applied [10] and 2D contours are obtained by defining an initial echo level in all XY-planes. The initial echo level is computed as a function of the mean echo level in the 3D scene. This level depends mainly on the grape variety but also on leaves density. For both Gamay and Black Manseng, initial echo level contours are of -80 dB. In a given XY-plane, only contours enclosing a surface between $10 \mathrm{~cm}^{2}$ and $100 \mathrm{~cm}^{2}$ are considered. This surface restriction removes from the clutter undesirable (very high or very low) echo levels. As a matter of fact surfaces that are smaller than $10 \mathrm{~cm}^{2}$ may generate false detections while surfaces higher than $100 \mathrm{~cm}^{2}$ may be due to multiple electromagnetic scatterers. The contour algorithm is iterated with higher echo levels until no contour encloses a surface larger than $100 \mathrm{~cm}^{2}$. 


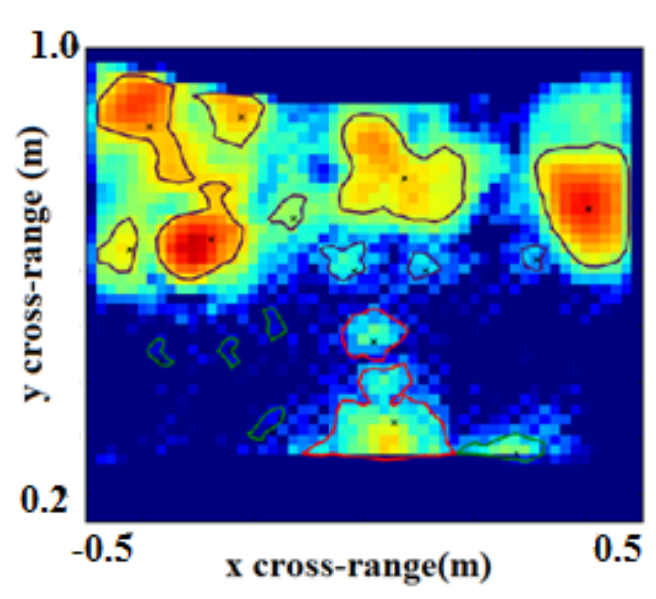

(a)

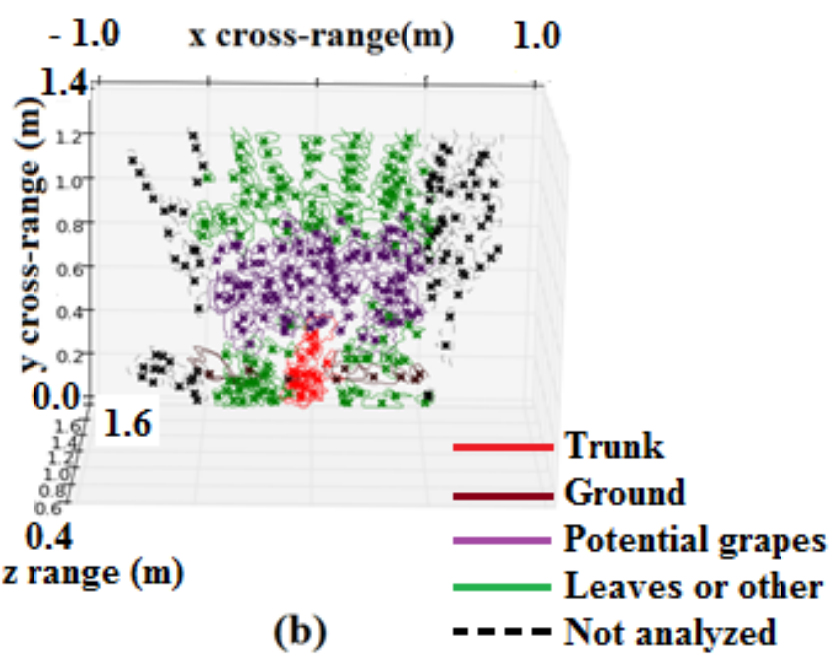

Fig 5. Echo level contours (a) in a cut-plane $\mathrm{z}=0.825 \mathrm{~m}$ and $(\mathrm{b})$ in the interrogated $3 \mathrm{D}$ space

Fig.5(a) shows contours computed from the previous Gamay grapevine plant in a cut-plane at $\mathrm{z}=0.825 \mathrm{~m}$. Echo levels are identified from their positions and red, green, purple, brown and black dashed contours are associated with respectively the trunk, leaves (or other), grapes, ground and not analyzed echoes. Contours of each XY-plane are combined in order to make a list of echoes in the interrogated 3D scene. These echoes are shown on Fig.5(b). For each echo different parameters are calculated: (a) its position (or barycenter), (b) its mean value, (c) its standard deviation value, (d) its maximal value, (e) its "shape" such as, e.g., the height, width and depth, and finally (f) its "volume" that is, the number of voxels contained within the contour multiplied by the volume resolution $s$ given by Eq.(1). Data associated with the purple contours in Fig.5(b) are potentially generated by the electromagnetic scattering of grapes and consequently, they must be analyzed in order to avoid eventual false detections of grapes. A new parameter to perform the echoes classification is defined here for the first time as follows:

$$
\chi=\frac{C_{\operatorname{Max}}}{\hat{C}}
$$

where $C_{M a x}$ denotes the maximal value of measured echo levels and designates the arithmetic mean of the echo levels. We call this parameter the spread factor. 3D contours with close spread factors have very similar distribution of echo levels. To highlight the physical meaning of this new parameter, Fig. 6 reports the computed values of $\chi$ for Gamay and for Black Manseng grapevine plants before and after grapes crop. For Gamay variety the number of contours such that $\chi>0.95$ is higher before the crop than after the crop. Moreover, for Black Manseng variety the number of contours such that $0.98>\chi>0.94$ is higher before the crop than after the crop. Consequently grapes variety are characterized by a specific spread factor which can be used for classification purpose.

Let $V_{E S T}=N_{E S T} \times s$ be an estimation of echo volume, where $N_{E S T}$ is the number of voxels for which $\chi \geq 0.95$ in case of Gamay variety (or for which $0.94<\chi<0.98$ in case of Black Manseng variety). Note that a correction factor must be applied to $V_{E S T}$ for each grapevine by determining the number of voxels in a region without grapes (that is, for example, at a height higher than $0.9 \mathrm{~m}$ ). Fig. 7 reports the estimator $V_{E S T}$ as a function of the grapes mass. The mass has been measured just after the crop and radar interrogation. We observe that $V_{E S T}$ increases linearly with the grapes mass with a slope of $0.17 \mathrm{~g} / \mathrm{cm}^{3}$ (the coefficient of determination is found to be $\mathrm{R}^{2}=0.947$ and a standard error of $0.02 \mathrm{~g}$ ). This convenient linear variation is obtained from detected grapes but also from 
undesirable scatterers in the 3D scene such as leaves and wood. The volume of grapes can be estimated by using a unique grapevine for calibration purpose. Assuming that volumetric mass density of grapes is known for a given variety, the physical volume of grapes can then be remotely derived directly from the computation of $V_{E S T}$.

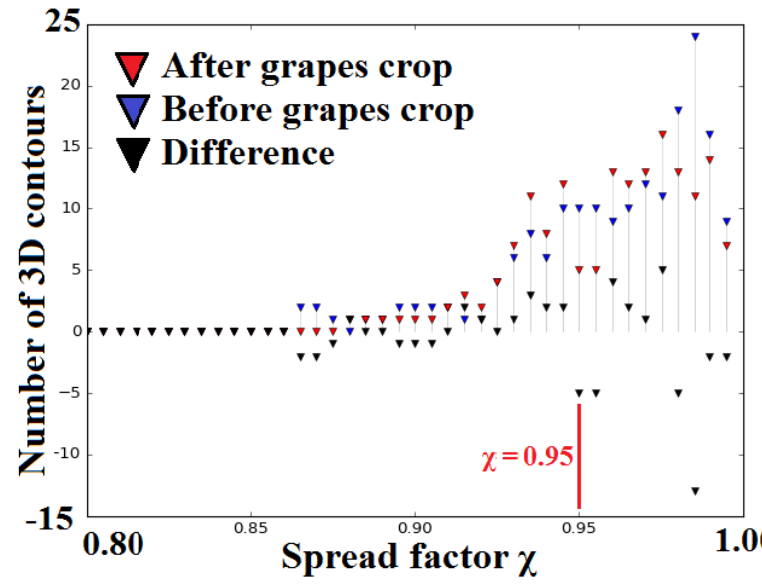

(a)

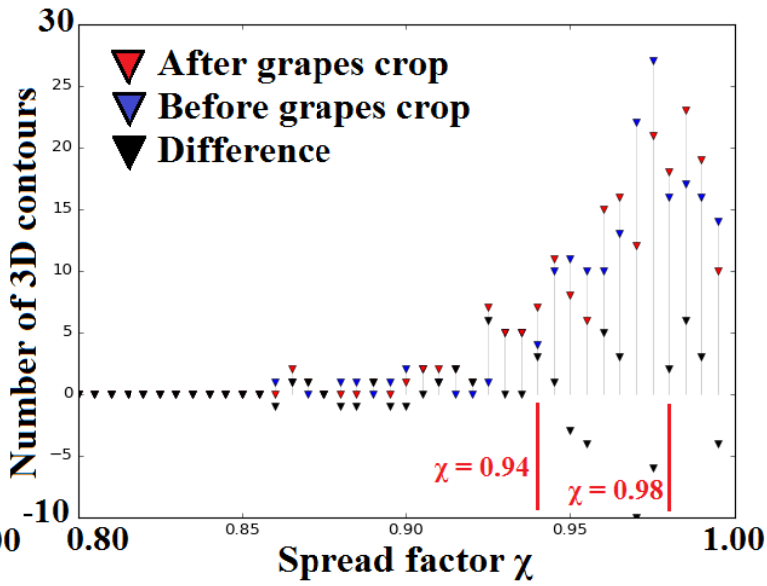

(b)

Fig 6. Number of 3D contours as a function of the so-called spread factor for (a) Gamay and (b) Black Manseng grape varieties after and before the crop.

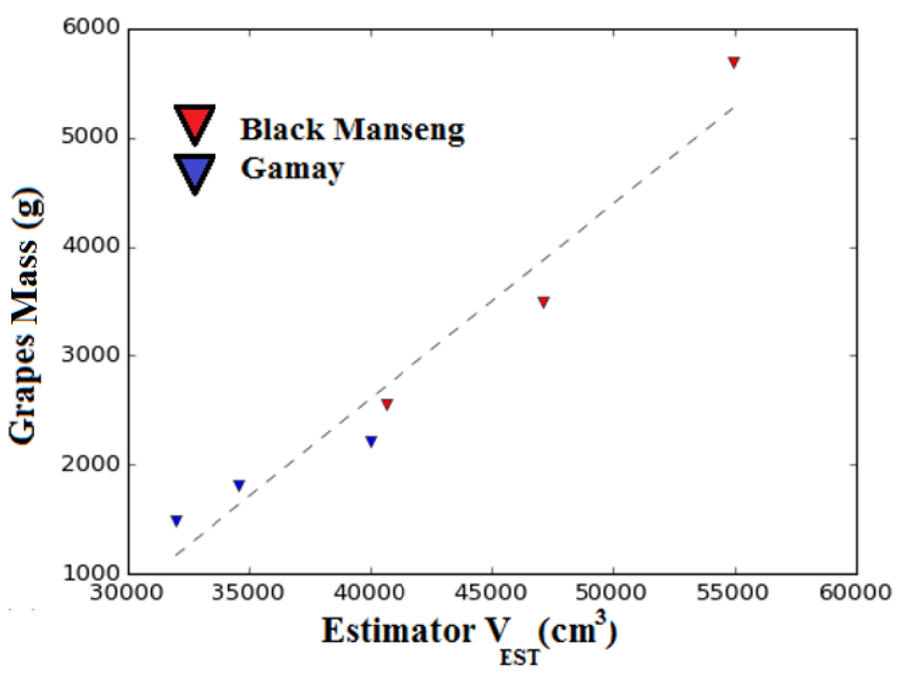

Fig 7. Grapes mass for three grapevines as a function of the estimator $\mathrm{V}_{\mathrm{EST}}$. The mass of grapes has been measured after crop and radar interrogation.

\section{Conclusion}

In this paper a solution to remotely estimate the volume of grapes is proposed by performing a $3 \mathrm{D}$ beam scanning of a ground-based $24 \mathrm{GHz}$ FMCW radar. A new parameter called the spread factor is defined in order to perform a classification of the measured 3D echo levels. By choosing the appropriate spread factor for a given grapevine variety, an estimator based on the number of voxels is computed for deriving the quantity of grapes (mass or volume if the volumetric mass 
density of grapes is known) in each grapevine, even if the grapes are hidden by leaves. This estimation has been applied with success to two grape varieties and to six grapevines. The sensitivity of the estimator is found to be $0.17 \mathrm{~g} / \mathrm{cm}^{3}$ for a linear model with $\mathrm{R}^{2}=0.947$ and a standard error of $0.02 \mathrm{~g}$. This model will be applied to numerous radar data for reaching the targeted accuracy $(<10 \%)$ of grapes volume estimation.

\section{Acknowledgement}

The authors wish to thank the French Technological Research Agency (Agence Nationale de la Recherche Technologique, France) for contribution in the financial support of this study.

\section{References}

[1] Johnson LF, Roczen DE, Youkhana SK, Nemani RR, Bosch DF. "Mapping vineyard leaf area with multispectral satellite imagery," Computers and Electronics in Agriculture. 2003;38:3344.

[2] F. André et al., "High-resolution imaging of a vineyard in south of France using ground penetrating radar and electromagnetic induction," Proceedings of the XIII International Conference on Ground Penetrating Radar, Lecce, 2010, pp. 1-8

[3] M. Larrain, A. R. Guesalaga, E. Agosin, "A Multipurpose Portable Instrument for Determining Ripeness in Wine Grapes Using NIR Spectroscopy," IEEE Transactions on Instrumentation and Measurement, vol. 57, no. 2, pp. 294-302, Feb. 2008.

[4] V. M. Gomes, A.M. Fernandes, A.Faia, P.Melo-Pinto,"Determination of sugar content in whole Port Wine grape berries combining hyperspectral imaging with neural networks methodologies", Computational Intelligence for Engineering Solutions (CIES) 2014 IEEE Symposium on, pp. 188-193, 2014.

[5] Bramley, R.G.V., "Progress in the Development of Precision Viticulture-Variation in Yield, Quality and Soil Properties in Contrasting Australian Vineyards. In Precision Tools for Improving Land Management," Currie, L.D., Loganathan, P., Eds.; Massey University: Palmerston North, New Zealand, 2001; pp. 25-43.

[6] A. Burini, G. Schiavon, D. Solimini, "Fusion of High Resolution Polarimetric SAR and Multi-Spectral Optical Data for Precision Viticulture," IGARSS 2008 - 2008 IEEE International Geoscience and Remote Sensing Symposium, Boston, MA, 2008, pp. III - 1000-III - 1003.

[7] L. Sun et al., "Daily mapping of Landsat-like LAI and correlation to grape yield," 2016 IEEE International Geoscience and Remote Sensing Symposium (IGARSS), Beijing, 2016, pp. 7157-7160.

[8] Sanz R., Rosell J.R., Llorens J., Gil E., Planas S., "Relationship between tree row LIDARvolume and leaf area density for fruit orchards and vineyards obtained with a LIDAR 3D Dynamic Measurement System," Agric. Forest Meteorol. 2013;171:153-162.

[9] P. Dolezel, P. Skrabanek, L. Gago, "Detection of grapes in natural environment using feedforward neural network as a classifier," 2016 SAI Computing Conference (SAI), London, 2016, pp. 1330-1334. 
[10] Lorensen, William and Harvey E. Cline. Marching Cubes: A High Resolution 3D Surface Construction Algorithm. Computer Graphics (SIGGRAPH 87 Proceedings) 21(4) July 1987, p. 163170)

Dominique Henry received the M.S. degree in electronical engineering from National Polytechnical Institute, Toulouse, France, in 2012. He is currently pursuing the Ph.D. degree in electronical engineering at Ovalie-Innovation, Auch, France. From 2013 to 2014, he was an engineer with the LAAS-CNRS, Toulouse, France. His research interest includes use of microwave radars for agricultural application, the remote sensing of passive sensors, development of radar interrogation techniques, microwave imaging and signal processing, and design of antennas for constrained environments

Hervé Aubert was born in Toulouse, France, in July 1966. He received the Eng. Dipl. in July 1989 and the Ph.D. degree (with high-honors) in January 1993, both in Electrical Engineering and both from the Institut National Polytechnique (INPT), Toulouse, France. Since February 2001 Hervé Aubert is Full Professor at INPT. He has joined the Laboratory for the Analysis and Architecture of Systems (LAAS), National Center for Scientific Research (CNRS), Toulouse, in February 2006. Since January 2015 he is the Head of the Micro- and Nano-systems for Wireless Communications Research Group at LAAS-CNRS.

Dr. Aubert has performed research works on integral-equation and variational methods applied to electromagnetic wave propagation and scattering. Currently his research activities involve the electromagnetic modelling of complex (multi-scale) structures, and the wireless electromagnetic sensors. He has authored or co-authored 75 papers in indexed journals and 200 communications in International Symposium Proceedings.

Dr. Aubert serves as an Associate Editor of Electronics Letters (since 2015) and he is a member of the editorial board of International Journal of Antennas and Propagation (since 2014) and of International Journal of Microwave Science and Technology (since 2010). Dr. Aubert was the General Chairman of the European Microwave Week in 2015 (Paris, France).

He was the Secretary of IEEE Antennas and Propagation French Chapter from 2009 to 2013, the Vice-Chairman of this Chapter from 2004 to 2009 and Secretary from 2001 to 2004. He is a senior member of IEEE (since 1999).

Thierry Véronèse was born in Grenoble, France, in May 1968. He received an engineer diploma and a pH.D degree from the National Institute of Applied Sciences of Toulouse specialized in industrial biotechnologies and food biochemistry. His carreer was mainly carried out in industrial and private organizations as researcher and project manager. He has been spending 10 years in developing and coordinating innovation projects on agriculture chains between academic research and industry on intersectorial operations (bio-energies, chemistry, health/cosmetics, agriculture/food and sensor industries) with cross-disciplinary approaches (agronomy, plant and industrial biotechnologies, green chemistry, digital and sensors technologies...) at regional, French and European levels. Since 2012, Dr Véronèse is the scientific manager of the company OvalieInnovation which is a subsidiary of two cooperative groups in the South-West of France. This company is involved in more than 10 collaborative research programs with academic research and other industrial companies from different sectors. One of its major items of the R\&D portfolio 
concerns the development of precision farming by the use of sensor and digital technologies vectorised by satellite, robots or drones... and combined with the more recent knowledge in agronomy. 